PROCEEDINGS OF THE

AMERICAN MATHEMATICAL SOCIETY

Volume 131, Number 5 , Pages 1481-1488

S 0002-9939(02)06654-6

Article electronically published on September 20, 2002

\title{
THE QUASILINEARIZATION METHOD ON AN UNBOUNDED DOMAIN
}

PAUL W. ELOE

(Communicated by Carmen C. Chicone)

\begin{abstract}
We apply a method of quasilinearization to a boundary value problem for an ordinary differential equation on an unbounded domain. A uniquely determined Green's function, which is integrable and of fixed sign, is employed. The hypotheses to apply the quasilinearization method imply uniqueness of solutions. The quasilinearization method generates a bilateral iteration scheme in which the iterates converge monotonically and quadratically to the unique solution.
\end{abstract}

In this paper, we shall apply a method of quasilinearization to the singular boundary value problem (BVP),

$$
\begin{gathered}
x^{\prime \prime}(t)+q(t) x(t)=f(t, x(t)), \quad t \in \mathbb{R}^{+}, \\
x(0)=x_{0}, \quad x(t) \text { bounded on } \mathbb{R}^{+},
\end{gathered}
$$

where $x_{0}$ is real, $f: \mathbb{R}^{+} \times \mathbb{R} \rightarrow \mathbb{R}$ is continuous, $q: \mathbb{R}^{+} \rightarrow \mathbb{R}^{-}$is continuous, and $q(t) \leq-c^{2}<0, t \in \mathbb{R}^{+}$, for some $c^{2}>0$. We model the singular BVP based on the work of Bebernes and Jackson [1].

The method of quasilinearization has recently been studied and extended extensively. It is generating a rich history beginning with the works by Bellman [2, 3]. Lakshmikantham, Leela, Vatsala, and many co-authors have extensively developed the method and have applied the method to a wide range of problems. We refer the reader to the recent work by Lakshmikantham and Vatsala 12 and the extensive bibliography found there. The method we produce here is modeled by the method developed by Lakshmikantham, Leela and McRae [11; this method is referred to as the improved generalized quasilinearization method. Analogous methods have been applied to two-point boundary value problems for ordinary differential equations and we refer the reader to the papers, $15,13,14,10,6,7,4$,

To our knowledge, this paper provides the first application of the quasilinearization method to singular boundary value problems on unbounded domains. Devi and Vatsala [5] have recently applied the method to a singular BVP on a bounded domain.

The method of quasilinearization for BVPs employs a delicate balance of upper and lower solution methods with monotone methods. Recently, Eloe, Grimm and

Received by the editors August 10, 2001 and, in revised form, December 11, 2001.

1991 Mathematics Subject Classification. Primary 34B40, 34A45.

Key words and phrases. Quasilinearization, quadratic convergence, upper and lower solutions, singular boundary value problems. 
Mashburn 8 developed both the monotone methods and the upper and lower solution methods that provide the supporting material. Before we develop the quasilinearization method here, we shall prove one result, Theorem 1 not proved by Eloe, Grimm and Mashburn [8]; we shall state without proof the other results we require from 8 . In Theorem 2 we shall develop the method of quasilinearization.

Throughout most of this paper (Lemmas 10 2. and 3 are exceptions) we shall assume that

$$
x_{1} \leq x_{2} \Longrightarrow f\left(t, x_{1}\right) \leq f\left(t, x_{2}\right), \quad t \in \mathbb{R}^{+} .
$$

We also require the definitions of upper and lower solutions for the BVP, (1), (2) .

$\beta$ is an upper solution of the BVP, (11), (2), if $\beta^{\prime \prime}$ is continuous on $\mathbb{R}^{+}$and

$$
\begin{gathered}
\beta^{\prime \prime}(t)+q(t) \beta(t) \leq f(t, \beta(t)), \quad t \in \mathbb{R}^{+}, \\
\beta(0) \geq x_{0}, \quad \beta(t) \text { bounded on } \mathbb{R}^{+},
\end{gathered}
$$

and $\alpha$ is a lower solution of the BVP, (1), (2), if $\alpha^{\prime \prime}$ is continuous on $\mathbb{R}^{+}$and

$$
\begin{gathered}
\alpha^{\prime \prime}(t)+q(t) \alpha(t) \geq f(t, \alpha(t)), \quad t \in \mathbb{R}^{+}, \\
\alpha(0) \leq x_{0}, \quad \alpha(t) \text { bounded on } \mathbb{R}^{+} .
\end{gathered}
$$

Theorem 1. Assume the monotonicity condition (3). Let $\alpha$ and $\beta$ denote a lower and an upper solution of the BVP, (1), (2), respectively. Then

$$
\alpha(t) \leq \beta(t), \quad t \in \mathbb{R}^{+} .
$$

Proof. Set $h=\alpha-\beta$ and note by the definition of upper and lower solutions that $h(0) \leq 0$. Assume for the sake of contradiction that $h$ is not non-positive on $\mathbb{R}^{+}$. Set

$$
\tau=\sup \{t \geq 0: h(t) \leq 0\} .
$$

Then $h(\tau)=0$ and $h^{\prime}(\tau) \geq 0$.

As a first case, assume there is some $t_{1}>\tau$ such that $h^{\prime}\left(t_{1}\right)<0$. Then there exists some $t_{0} \in\left(\tau, t_{1}\right)$ such that $h\left(t_{0}\right)$ is a maximum value of $h$ on $\left[\tau, t_{1}\right]$. Thus, $h\left(t_{0}\right)>0, h^{\prime}\left(t_{0}\right)=0$, and $h^{\prime \prime}\left(t_{0}\right) \leq 0$. By the definition of upper and lower solutions, and an application of (3),

$$
h^{\prime \prime}\left(t_{0}\right) \geq f\left(t_{0}, \alpha\left(t_{0}\right)\right)-f\left(t_{0}, \beta\left(t_{0}\right)\right)-q\left(t_{0}\right) h\left(t_{0}\right)>0 .
$$

This is a contradiction and so, for the second case, assume $h^{\prime}(t) \geq 0, t \geq \tau$. By the definition of $\tau, h(\tau+1)>0$. Thus, there exists $c \in(\tau, \tau+1)$ such that $h^{\prime}(c)>0$. It follows as in the development of (5) that if $h(t) \geq 0$, then $h^{\prime \prime}(t) \geq 0$. Thus, $h^{\prime \prime}(t) \geq 0$ on $(\tau, \infty)$ and so, $h^{\prime}$ is nondecreasing on $(\tau, \infty)$. In particular,

$$
h^{\prime}(t) \geq h^{\prime}(c)>0, \quad t \in[c, \infty),
$$

which implies that $h$ is unbounded. This contradicts the definition of upper and lower solutions of the BVP, (1), (2) and completes the proof.

Corollary 1. Under the hypotheses of Theorem 11 solutions of the BVP, (11), (2), are unique.

Lemmas 1, 2] and 3] have all been obtained in [8]. Theorem 4.2 is stated incorrectly in $[8]$ and is stated correctly here in Lemma 1 . 
Lemma 1. Assume $f: \mathbb{R}^{+} \times \mathbb{R} \rightarrow \mathbb{R}$ is continuous and for each $M>0$, there exists $f_{M}: \mathbb{R}^{+} \rightarrow \mathbb{R}^{+}$which is bounded and continuous and such that

$$
|f(s, x)| \leq f_{M}(s), \quad|x| \leq M .
$$

Assume $q: \mathbb{R}^{+} \rightarrow \mathbb{R}^{-}$is continuous such that $q(t) \leq-c^{2}<0$ for some $c^{2}>$ 0 . Assume there exist $\alpha$ and $\beta$, lower and upper solutions of the BVP, (11), (2), respectively, such that $\alpha$ and $\beta$ satisfy (4). Then, there exists a solution, $x$, of the $B V P$, (1), (2), satisfying

$$
\alpha(t) \leq x(t) \leq \beta(t), \quad t \in \mathbb{R}^{+} .
$$

Lemma 2. If $-q(t) \geq c^{2}>0$, then there exists a unique solution, $p_{0}(t)$, of the $B V P$,

$$
x^{\prime \prime}(t)+q(t) x(t)=0, \quad t \in \mathbb{R}^{+},
$$

satisfying the boundary conditions, (2).

Lemma 3. Assume $f: \mathbb{R}^{+} \times \mathbb{R} \rightarrow \mathbb{R}$ is continuous and for each $M>0$, there exists $f_{M}: \mathbb{R}^{+} \rightarrow \mathbb{R}^{+}$which is bounded and continuous and such that

$$
|f(s, x)| \leq f_{M}(s), \quad|x| \leq M .
$$

Assume $q: \mathbb{R}^{+} \rightarrow \mathbb{R}^{-}$is continuous such that $q(t) \leq-c^{2}<0$ for some $c^{2}>0$. Then there exists a uniquely defined Green's function, $G(t, s)$, defined on $\mathbb{R}^{+} \times \mathbb{R}^{+}$ such that $x$ is a solution of the BVP, (11), (2), if, and only if, $x$ is bounded and continuous on $\mathbb{R}^{+}$and

$$
x(t)=p_{0}(t)+\int_{0}^{\infty} G(t, s) f(s, x(s)) d s, \quad t \in \mathbb{R}^{+} .
$$

The Green's function satisfies the sign property

$$
G(t, s)<0,(t, s) \in(0, \infty) \times(0, \infty)
$$

and the integrability property

$$
\int_{0}^{\infty}|G(t, s)| d s \leq 1 / c^{2} .
$$

We now develop the quasilinearization method for the BVP, (1), (2).

Theorem 2. Assume $f$ satisfies the monotonicity condition (3). Assume $f_{x}$ and $f_{x x}$ exist and are continuous on $\mathbb{R}^{+} \times \mathbb{R}$. Assume $f: \mathbb{R}^{+} \times \mathbb{R} \rightarrow \mathbb{R}$ is continuous and for each $M>0$, there exists $f_{M}: \mathbb{R}^{+} \rightarrow \mathbb{R}^{+}$which is bounded and continuous and such that

$$
|f(s, x)| \leq f_{M}(s), \quad\left|f_{x}(s, x)\right| \leq f_{M}(s), \quad|x| \leq M .
$$

Assume $q: \mathbb{R}^{+} \rightarrow \mathbb{R}^{-}$is continuous such that $q(t) \leq-c^{2}<0$ for some $c^{2}>$ 0 . Assume there exists $\phi(t, x)$, defined on $\mathbb{R}^{+} \times \mathbb{R}$, such that $\phi, \phi_{x}$, and $\phi_{x x}$ are continuous on $\mathbb{R}^{+} \times \mathbb{R}$ and such that

$$
\phi_{x x}(t, x)>0, \quad(t, s) \in \mathbb{R}^{+} \times \mathbb{R} .
$$

Moreover, assume there exists $\phi_{M}: \mathbb{R}^{+} \rightarrow \mathbb{R}^{+}$which is bounded and continuous and such that

$$
|\phi(s, x)| \leq \phi_{M}(s),\left|\phi_{x}(s, x)\right| \leq \phi_{M}(s),\left|\phi_{x x}(s, x)\right| \leq \phi_{M}(s), \quad|x| \leq M .
$$

Set $\phi=F-f$; we shall require that

$$
F_{x x}(t, x) \geq 0, \quad(t, s) \in \mathbb{R}^{+} \times \mathbb{R} .
$$


Assume $\alpha_{0}$ and $\beta_{0}$, are lower and upper solutions of the $B V P$, (11), (2), respectively. Then there exist monotone sequences, $\left\{\alpha_{n}\right\}$ and $\left\{\beta_{n}\right\}$, that are solutions of linear differential equations and that converge in the space of bounded continuous functions on $\mathbb{R}^{+}$to the unique solution of the BVP, (11), (2). Moreover, the convergence is quadratic.

Proof. First apply Theorem 1 and then Lemma 1 to note that there exists a unique solution, $x$, of the BVP, (1), (2), satisfying

$$
\alpha_{0}(t) \leq x(t) \leq \beta_{0}(t), \quad t \in \mathbb{R}^{+} .
$$

By (7), it follows that

(8) $f(t, x) \geq f(t, y)+F_{x}(t, y)(x-y)-(\phi(t, x)-\phi(t, y)), \quad(t, x, y) \in \mathbb{R}^{+} \times \mathbb{R}^{2}$.

$t \in \mathbb{R}^{+}, x, y \in \mathbb{R}$.

Define two truncations for the nonlinear term $f$. Define

$$
g\left(t, x ; \alpha_{0}, \beta_{0}\right)=f\left(t, \alpha_{0}(t)\right)+F_{x}\left(t, \beta_{0}(t)\right)\left(x-\alpha_{0}(t)\right)-\left(\phi(t, x)-\phi\left(t, \alpha_{0}(t)\right)\right)
$$

and

$$
G\left(t, x ; \beta_{0}\right)=f\left(t, \beta_{0}(t)\right)+F_{x}\left(t, \beta_{0}(t)\right)\left(x-\beta_{0}(t)\right)-\left(\phi(t, x)-\phi\left(t, \beta_{0}(t)\right)\right) .
$$

For the sake of exposition, we may suppress explicit dependence on $t$ in some of the algebraic expressions below. Note that since $\phi_{x x}(t, x)>0$, it follows that

$$
g_{x x}(t, x ; u, v)<0, \quad G_{x x}(t, x ; v)<0, \quad(t, x) \in \mathbb{R}^{+} \times \mathbb{R} .
$$

Thus,

$$
g(t, x ; u, v) \geq g(t, y ; u, v)+g_{x}(t, x ; u, v)(x-y), \quad(t, x, y) \in \mathbb{R}^{+} \times \mathbb{R}^{2},
$$

and

$$
G(t, x ; u) \geq G(t, y ; u)+G_{x}(t, x ; u)(x-y), \quad(t, x, y) \in \mathbb{R}^{+} \times \mathbb{R}^{2} .
$$

Define further linear truncations, $h$ and $H$, by

$$
\begin{gathered}
h\left(t, x ; \alpha_{0}, \beta_{0}\right)=g\left(t, \alpha_{0}(t) ; \alpha_{0}, \beta_{0}\right)+g_{x}\left(t, \alpha_{0}(t) ; \alpha_{0}, \beta_{0}\right)\left(x-\alpha_{0}\right), \\
H\left(t, x ; \alpha_{0}, \beta_{0}\right)=G\left(t, \beta_{0}(t) ; \beta_{0}\right)+G_{x}\left(t, \alpha_{0}(t) ; \beta_{0}\right)\left(x-\beta_{0}\right),
\end{gathered}
$$

and consider two additional linear BVPs,

$$
x^{\prime \prime}(t)+q(t) x(t)=h\left(t, x ; \alpha_{0}, \beta_{0}\right), \quad t \in \mathbb{R}^{+},
$$

with the boundary conditions, (2), and

$$
x^{\prime \prime}(t)+q(t) x(t)=H\left(t, x ; \alpha_{0}, \beta_{0}\right), \quad t \in \mathbb{R}^{+},
$$

with the boundary conditions, (2). Because of the boundedness assumptions on $f$, $f_{x}, \phi$, and $\phi_{x}$, Lemma 1 can apply to each of the BVPs, (11), (2), and (12), (2).

We shall outline the first portion of the proof before providing the technical details. We have already noted that $\alpha_{0}(t) \leq \beta_{0}(t), t \in \mathbb{R}^{+}$. Show that $\alpha_{0}$ and $\beta_{0}$ are lower and upper solutions for the truncated BVP, (11), (2); apply Lemma 1 and obtain a solution, $\alpha_{1}$, of the BVP, (11), (2), satisfying

$$
\alpha_{0}(t) \leq \alpha_{1}(t) \leq \beta_{0}(t), \quad t \in \mathbb{R}^{+} .
$$

Similarly, show that $\alpha_{0}$ and $\beta_{0}$ are lower and upper solutions for the truncated BVP, (12), (2); apply Lemma 1 and obtain a solution, $\beta_{1}$, of the BVP, (12), (2), satisfying

$$
\alpha_{0}(t) \leq \beta_{1}(t) \leq \beta_{0}(t), \quad t \in \mathbb{R}^{+} .
$$


Now, show that $\alpha_{1}$ and $\beta_{1}$ are lower and upper solutions for the original BVP, (1), (2); apply Theorem 1 and obtain

$$
\alpha_{0}(t) \leq \alpha_{1}(t) \leq \beta_{1}(t) \leq \beta_{0}(t), \quad t \in \mathbb{R}^{+} .
$$

Let us first obtain (13). Since

$$
h\left(t, \alpha_{0} ; \alpha_{0}, \beta_{0}\right)=g\left(t, \alpha_{0} ; \alpha_{0}, \beta_{0}\right)=f\left(t, \alpha_{0}\right),
$$

$\alpha_{0}$ is clearly a lower solution of the BVP, (11), (21). As for $\beta_{0}$, apply (8) and (91) to obtain

$$
\begin{aligned}
f\left(t, \beta_{0}(t)\right) & \leq f\left(t, \alpha_{0}(t)\right)-F_{x}\left(t, \beta_{0}(t)\right)\left(\alpha_{0}(t)-\beta_{0}(t)\right) \\
& +\left(\phi\left(t, \alpha_{0}(t)\right)-\phi\left(t, \beta_{0}(t)\right)\right)=g\left(t, \beta_{0}(t) ; \alpha_{0}, \beta_{0}\right) \\
& \leq g\left(t, \alpha_{0}(t) ; \alpha_{0}, \beta_{0}\right)+g_{x}\left(t, \alpha_{0}(t) ; \alpha_{0}, \beta_{0}\right)\left(\beta_{0}-\alpha_{0}\right) \\
& =h\left(t, \beta_{0} ; \alpha_{0}, \beta_{0}\right) .
\end{aligned}
$$

Thus, $\beta_{0}$ is an upper solution of the BVP, (11), (2). Apply Lemma 1 and obtain the existence of a solution, $\alpha_{1}$, of the BVP, (11), (22), satisfying (13).

To obtain (14), $\beta_{0}$ is now clearly an upper solution of the BVP, (12), (2). As for $\alpha_{0}$, apply (8) and (10) to obtain

$$
\begin{aligned}
f\left(t, \alpha_{0}(t)\right) & \geq f\left(t, \beta_{0}(t)\right)+F_{x}\left(t, \beta_{0}(t)\right)\left(\alpha_{0}(t)-\beta_{0}(t)\right) \\
& -\left(\phi\left(t, \alpha_{0}\right)-\phi\left(t, \beta_{0}\right)\right)=G\left(t, \alpha_{0}(t) ; \beta_{0}\right) \\
& \geq G\left(t, \beta_{0}(t) ; \beta_{0}\right)+G_{x}\left(t, \alpha_{0}(t) ; \beta_{0}\right)\left(\alpha_{0}-\beta_{0}\right) \\
& =H\left(t, \beta_{0} ; \alpha_{0}, \beta_{0}\right) .
\end{aligned}
$$

Thus, $\alpha_{0}$ is a lower solution of the BVP, (12), (2). Apply Lemma 1 and obtain the existence of a solution, $\beta_{1}$, of the BVP, (12), (21), satisfying (14).

To complete the first part of the argument, we show that $\alpha_{1}$ and $\beta_{1}$ are lower and upper solutions, respectively of the original BVP, (1), (2) . First, consider, $\alpha_{1}$. Apply (9), (8) and note that $F_{x x} \geq 0$ implies $F_{x}$ is increasing in $x$ to obtain

$$
\begin{aligned}
h\left(t, \alpha_{1} ; \alpha_{0}, \beta_{0}\right) & =g\left(t, \alpha_{0} ; \alpha_{0}, \beta_{0}\right)+g_{x}\left(t, \alpha_{0} ; \alpha_{0}, \beta_{0}\right)\left(\alpha_{1}-\alpha_{0}\right) \\
& \geq g\left(t, \alpha_{1} ; \alpha_{0}, \beta_{0}\right) \\
& =f\left(t, \alpha_{0}\right)+F_{x}\left(t, \beta_{0}\right)\left(\alpha_{1}(t)-\alpha_{0}(t)\right)-\left(\phi\left(t, \alpha_{1}\right)-\phi\left(t, \alpha_{0}\right)\right) \\
& \geq f\left(t, \alpha_{0}\right)+F_{x}\left(t, \alpha_{1}\right)\left(\alpha_{1}(t)-\alpha_{0}(t)\right)-\left(\phi\left(t, \alpha_{1}\right)-\phi\left(t, \alpha_{0}\right)\right) \\
& \geq f\left(t, \alpha_{1}(t)\right) .
\end{aligned}
$$

Thus, $\alpha_{1}$ is a lower solution of the BVP, (1), (2). To see that $\beta_{1}$ is an upper solution of the BVP, (11), (2), employ (10) and $G_{x}$ decreasing to obtain

$$
\begin{aligned}
H\left(t, \beta_{1} ; \alpha_{0}, \beta_{0}\right) & =G\left(t, \beta_{0} ; \beta_{0}\right)+G_{x}\left(t, \alpha_{0} ; \beta_{0}\right)\left(\beta_{1}-\beta_{0}\right) \\
& \leq G\left(t, \beta_{1} ; \beta_{0}\right)+\left(G_{x}\left(t, \alpha_{0} ; \beta_{0}\right)-G_{x}\left(t, \beta_{1} ; \beta_{0}\right)\right)\left(\beta_{1}-\beta_{0}\right) \\
& \leq G\left(t, \beta_{1} ; \beta_{0}\right) \leq f\left(t, \beta_{1}\right) .
\end{aligned}
$$

The next step of the proof is inductive and we outline the details. Define inductively

$$
\begin{gathered}
g\left(t, x ; \alpha_{n}, \beta_{n}\right)=f\left(t, \alpha_{n}(t)\right)+F_{x}\left(t, \beta_{n}(t)\right)\left(x-\alpha_{n}\right)-\left(\phi(t, x)-\phi\left(t, \alpha_{n}\right)\right), \\
G\left(t, x ; \beta_{n}\right)=f\left(t, \beta_{n}(t)\right)+F_{x}\left(t, \beta_{n}(t)\right)\left(x-\beta_{n}\right)-\left(\phi(t, x)-\phi\left(t, \beta_{n}\right)\right), \\
h\left(t, x ; \alpha_{n}, \beta_{n}\right)=g\left(t, \alpha_{n} ; \alpha_{n}, \beta_{n}\right)+g_{x}\left(t, \alpha_{n} ; \alpha_{n}, \beta_{n}\right)\left(x-\alpha_{n}\right),
\end{gathered}
$$


and

$$
H\left(t, x ; \alpha_{n}, \beta_{n}\right)=G\left(t, \beta_{n} ; \beta_{n}\right)+G_{x}\left(t, \alpha_{n} ; \beta_{n}\right)\left(x-\beta_{n}\right) .
$$

At each step, we have that $\alpha_{n}$ and $\beta_{n}$ are lower and upper solutions, respectively, of the BVP, (1), (2), so

$$
\alpha_{n}(t) \leq \beta_{n}(t), \quad t \in \mathbb{R}^{+},
$$

by Theorem 1. Show that $\alpha_{n}$ and $\beta_{n}$ are lower and upper solutions, respectively, of a BVP,

$$
x^{\prime \prime}(t)+q(t) x(t)=h\left(t, x(t) ; \alpha_{n}, \beta_{n}\right), \quad t \in \mathbb{R}^{+},
$$

satisfying the boundary conditions, (2), and apply Lemma 1 to obtain the existence of a solution, $\alpha_{n+1}$, of this BVP satisfying

$$
\alpha_{n}(t) \leq \alpha_{n+1}(t) \leq \beta_{n}(t), \quad t \in \mathbb{R}^{+} .
$$

Similarly, obtain the existence of a corresponding solution, $\beta_{n+1}$, of the BVP,

$$
x^{\prime \prime}(t)+q(t) x(t)=H\left(t, x(t) ; \alpha_{n}, \beta_{n}\right), \quad t \in \mathbb{R}^{+},
$$

satisfying the boundary conditions, (2); in particular,

$$
\alpha_{n}(t) \leq \beta_{n+1}(t) \leq \beta_{n}(t), \quad t \in \mathbb{R}^{+} .
$$

Finally, show that $\alpha_{n+1}$ and $\beta_{n+1}$ are lower and upper solutions, respectively, of the original BVP, (1), (2), and apply Theorem 1 to obtain

$$
\alpha_{n}(t) \leq \alpha_{n+1}(t) \leq \beta_{n+1}(t) \leq \beta_{n}(t), \quad t \in \mathbb{R}^{+} .
$$

Due to the monotonicity and boundedness, there exists $\alpha$ such that $\left\{\alpha_{n}\right\}$ converges uniformly to $\alpha$ on compact subsets of $\mathbb{R}^{+}$. Moreover,

$$
h\left(t, \alpha_{n+1} ; \alpha_{n}, \beta_{n}\right) \rightarrow f(t, \alpha) .
$$

It follows by Lemma 3 and the dominated convergence theorem that

$$
\alpha(t)=p_{0}(t)+\int_{0}^{\infty} G(t, s) f(s, \alpha(s)) d s, \quad t \in \mathbb{R}^{+} ;
$$

in particular, by Lemma 3, $\alpha=x$, the unique solution of the BVP, (11), (2). Similarly, $\beta=x$, the unique solution of the BVP, (11), (21).

We close the proof by showing the convergence is quadratic. We show the details for the convergence in $\beta_{n}$; the details for the the convergence in $\alpha_{n}$ are analogous. Let $x$ denote the unique solution of the BVP, (11), (2). Set,

$$
r_{n}=\beta_{n}-x \geq 0, \quad p_{n}=x-\alpha_{n} \geq 0, \quad e_{n}=\max \left\{\left\|r_{n}\right\|,\left\|p_{n}\right\|\right\},
$$

where $\|\cdot\|$ denotes the supremum norm on bounded continuous functions on $\mathbb{R}^{+}$.

With repeated applications of the mean value theorem, there exist

$$
x(t) \leq c_{n}(t) \leq d_{n}(t) \leq \beta_{n}(t)
$$


such that

$$
\begin{aligned}
r_{n+1}^{\prime \prime}(t) & +q(t) r_{n+1}(t)=\left(\beta_{n+1}^{\prime \prime}(t)+q(t) \beta_{n+1}(t)\right)-\left(x^{\prime \prime}(t)+q(t) x(t)\right) \\
& =H\left(t, \beta_{n+1} ; \alpha_{n}, \beta_{n}\right)-f(t, x) \\
& =f\left(t, \beta_{n}\right)+\left(F_{x}\left(t, \beta_{n}\right)-\phi_{x}\left(t, \alpha_{n}\right)\right)\left(\beta_{n+1}-\beta_{n}\right)-f(t, x) \\
& =f_{x}\left(t, c_{n}\right)\left(\beta_{n}-x\right)+\left(F_{x}\left(t, \beta_{n}\right)-\phi_{x}\left(t, \alpha_{n}\right)\right)\left(\beta_{n+1}-\beta_{n}\right) \\
& \geq f_{x}\left(t, c_{n}\right)\left(\beta_{n}-x\right)+\left(F_{x}\left(t, \beta_{n}\right)-\phi_{x}\left(t, c_{n}\right)\right)\left(\beta_{n+1}-\beta_{n}\right) \\
& =f_{x}\left(t, c_{n}\right)\left(\beta_{n}-x\right)+\left(F_{x}\left(t, \beta_{n}\right)-\phi_{x}\left(t, c_{n}\right)\right)\left(\beta_{n+1}-x\right) \\
& +\left(\phi_{x}\left(t, c_{n}(t)\right)-F_{x}\left(t, \beta_{n}(t)\right)\right)\left(\beta_{n}-x\right) \\
& =\left(F_{x}\left(t, c_{n}(t)\right)-F_{x}\left(t, \beta_{n}(t)\right)\right) r_{n}(t)+\left(F_{x}\left(t, \beta_{n}\right)-\phi_{x}\left(t, c_{n}\right)\right) r_{n+1}(t) \\
& \geq\left(F_{x}\left(t, c_{n}(t)\right)-F_{x}\left(t, \beta_{n}(t)\right)\right) r_{n}(t)+\left(F_{x}\left(t, c_{n}\right)-\phi_{x}\left(t, c_{n}\right)\right) r_{n+1}(t) \\
& \geq F_{x x}\left(t, d_{n}(t)\right)\left(c_{n}(t)-\beta_{n}(t)\right) r_{n}(t)+f_{x}\left(t, c_{n}\right) r_{n+1} \geq-M\left\|e_{n}\right\|^{2}
\end{aligned}
$$

for some $M>0$.

Finally, note that since $\beta_{n+1}$ and $x$ are bounded and are solutions of $x^{\prime \prime}(t)+$ $q(t) x(t)=H\left(t, x ; \alpha_{n}, \beta_{n}\right)$ and (1), respectively, it follows that $r_{n+1}^{\prime \prime}$ is bounded. Thus, $r_{n+1}$ is the unique solution of the BVP,

$$
x^{\prime \prime}(t)=r_{n+1}^{\prime \prime}(t)+q(t) r_{n+1}(t), \quad t \in \mathbb{R}^{+},
$$

satisfying the boundary conditions,

$$
x(0)=0, \quad x(t) \text { bounded on } \mathbb{R}^{+} ;
$$

in particular, by Lemma 3

$$
\begin{aligned}
0 \leq r_{n+1}(t) & =\int_{0}^{\infty} G(t, s)\left(r_{n+1}^{\prime \prime}(s)+q(s) r_{n+1}(s)\right) d s \\
& \leq M \int_{0}^{\infty}|G(t, s)| d s\left\|r_{n}\right\|^{2} \leq M\left\|r_{n}\right\|^{2} / c^{2}
\end{aligned}
$$

Remark 1. If $f_{x x} \geq 0$, and sufficiently bounded, then $F=f$ and $\phi=0$ is sufficient. Conditions other than the convexity condition, (7), can be employed [14]. For example, if $f_{x x} \leq 0$, and sufficiently bounded, take $F=f$ and $\phi=0$. Define truncations, $g$ and $G$, by

$$
\left.g\left(t, x ; \alpha_{0}\right)=f\left(t, \alpha_{0}(t)\right)+F_{x}\left(t, \alpha_{0}(t)\right)\left(x-\alpha_{0}(t)\right)\right)
$$

and

$$
G\left(t, x ; \beta_{0}, \alpha_{0}\right)=f\left(t, \beta_{0}(t)\right)+F_{x}\left(t, \alpha_{0}(t)\right)\left(x-\beta_{0}(t)\right) .
$$

See 9.

Remark 2. An analogous theorem to Theorem 2 can be stated and proved where one obtains quadratic convergence but the iterates are solutions of nonlinear equations. One employs $g$ and $G$ in the truncated BVPs instead of $h$ and $H$. Similar details are presented in 11], [10] or 12] for example. In the statement of the theorem, one would require $F$ to be convex and satisfy all the boundedness hypotheses; one would not need to require these conditions on $\phi$ or $f_{x}$. For this case, $F(t, x)=e^{-x} /\left(1+t^{2}\right)$ suffices and the hypotheses of the theorem are weakened.

The author thanks the referee for useful and insightful observations. 


\section{REFERENCES}

[1] J. W. Bebernes and L. K. Jackson, Infinite interval boundary value problems for $y^{\prime \prime}=f(t, y)$, Duke Math. J., 34 (1967), 39-47. MR 34:6205

[2] R. Bellman, Methods of Nonlinear Analysis, Vol. II Academic Press, New York, 1973. MR 52:2305

[3] R. Bellman and R. Kalaba, Quasilinearization and Nonlinear Boundary Value Problems, Elsevier, New York, 1965. MR 31:2828

[4] A. Cabada, J. Nieto and R. Pita-da-Viega, A note on rapid convergence of approximate solutions for an ordinary Dirichlet problem, Dynam. Contin. Discrete Impuls. Systems, 4 (1998), 23-30. MR 98k:34020

[5] J. V. Devi and A. S. Vatsala, Quasilinearization for second order singular boundary value problems with solutions in weighted spaces, J. Korean Math. Soc., 37 (2000), 823-833.

[6] V. Doddaballapur and P. W. Eloe, Monotone and quadratic convergence of approximate solutions of ordinary differential equations with impulse, Comm. Appl. Analysis, 2 (1998), 373-382. MR 99d:34021

[7] V. Doddaballapur, P. W. Eloe and Y. Zhang, Quadratic convergence of approximate solutions of two-point boundary value problems with impulse, Electronic J. Differential Equations, Conf. 01, (1997), 81-95. MR 99m:34019

[8] P. W. Eloe, L. J. Grimm and J. Mashburn, A boundary value problem on an unbounded domain, Differential Equations and Dynamical Systems, 8 (2000), 125-140.

[9] P. W. Eloe and S. G. Hristova, Application of the method of quasilinearization for the initial value problem for systems of impulsive differential equations, preprint.

[10] P. W. Eloe and Y. Zhang, A quadratic monotone iteration scheme for two-point boundary value problems for ordinary differential equations, Nonlinear Analysis, 33 (1998), 443-453. MR 99e:34015

[11] V. Lakschmikantham, S. Leela, and F. A. McRae, Improved generalized quasilinearization (GQL) method, Nonlinear Analysis, 24 (1995), 1627-1637. MR 96a:34023

[12] V. Lakschmikantham and A. S. Vatsala, Generalized Quasilinearization for Nonlinear Problems, Kluwer Academic Publishers, Dordrecht, 1998. MR 99k:34013

[13] R. N. Mohapatra, K. Vajravelu and Y. Yin, Generalized quasilinearization method for second order boundary value problem, Nonlinear Analysis, 36 (1999), 799-806. MR 2000f:34038

[14] J. Nieto, Generalized quasilinearization method for a second order differential equation with Dirichlet boundary conditions, Proc. Amer. Math. Soc., 125 (1997), 2599-2604. MR 97j:34015

[15] N. Shahzad and A. S. Vatsala, An extension of the method of generalized quasilinearization for second order boundary value problems, Applicable Analysis, 58 (1995), 77-83. MR 97a:34063

Department of Mathematics, University of Dayton, Dayton, Ohio 45469-2316

E-mail address: Paul.Eloe@notes.udayton.edu 